\title{
Exchange and correlation effects in the relaxation of hot electrons in noble metals
}

\author{
I. G. Gurtubay, ${ }^{1}$ J. M. Pitarke, $, 1,2$ and P. M. Echenique 2,3 \\ ${ }^{1}$ Materia Kondentsatuaren Fisika Saila, Zientzi Fakultatea, Euskal Herriko Unibertsitatea, 644 Posta kutxatila, E-48080 Bilbo, \\ Basque Country, Spain \\ ${ }^{2}$ Donostia International Physics Center (DIPC) and Centro Mixto CSIC-UPV/EHU, Manuel de Lardizabal Pasealekua, \\ E-20018 Donostia, Basque Country, Spain \\ ${ }^{3}$ Materialen Fisika Saila, Kimika Fakultatea, Euskal Herriko Unibertsitatea, 1071 Posta kutxatila, E-20018 Donostia, \\ Basque Country, Spain
}

(Received 9 February 2004; published 17 June 2004)

\begin{abstract}
We report extensive first-principles calculations of the inelastic lifetime of low-energy electrons in the noble metals $\mathrm{Cu}, \mathrm{Ag}$, and $\mathrm{Au}$. The quasiparticle self-energy is computed with full inclusion of exchange and correlation (XC) effects, in the framework of the $G W \Gamma$ approximation of many-body theory. Although exchange and correlation may considerably reduce both the screening and the bare interaction of hot electrons with the Fermi gas, these corrections have opposite signs. Our results indicate that the overall effect of short-range XC is small and $G W \Gamma$ linewidths are close to their XC-free $G^{0} W^{0}$ counterparts, as occurs in the case of a free-electron gas.
\end{abstract}

DOI: $10.1103 /$ PhysRevB.69.245106

PACS number(s): 71.45.Gm, 78.47.+p

\section{INTRODUCTION}

Relaxation lifetimes of excited electrons with energies larger than $1 \mathrm{eV}$ are mainly dominated by electron-electron $(e-e)$ inelastic interactions of the excited electron with the electrons in the valence bands of the solid. The inelastic lifetime of these so-called hot electrons has been investigated for many years on the basis of the free-electron gas (FEG) or jellium description of the solid, ${ }^{1-3}$ in which a homogeneous assembly of interacting electrons is assumed to be immersed in a uniform positive background. Nonetheless, timeresolved (TR) two-photon photoemission (2PPE) experiments ${ }^{4-11}$ and ballistic electron-emission spectroscopy (BEES) (Ref. 12) have shown that band-structure effects play a key role in the decay mechanism.

The first theoretical investigations of the hot-electron dynamics which take into account explicitly the band structure of the solid were performed only a few years ago. ${ }^{13,14}$ Since then, first-principles calculations of the $e$-e scattering have been reported for simple, ${ }^{15}$ noble, ${ }^{16-18}$ and transition metals. ${ }^{19-21}$ Nevertheless, all existing calculations have been performed within the $G^{0} W^{0}$ approximation of many-body theory, ${ }^{22}$ with no inclusion of exchange and correlation (XC) effects.

In this work, we go beyond the $G^{0} W^{0}$ approximation by including XC effects both in the description of the dynamical screening of the many-electron system [we go beyond the random-phase approximation (RPA) in the evaluation of the screened interaction $W]$ and in the expansion of the electron self-energy in terms of $W$. This is the $G W \Gamma$ approximation, ${ }^{23,24}$ which treats on the same footing XC effects between pairs of electrons within the Fermi sea (screening electrons) and between the hot excited electron and the Fermi sea. Mahan and Sernelius ${ }^{23}$ showed that the inclusion of the same vertex function in the screened interaction and the numerator of the self-energy yields results for the bandwidth of a homogeneous electron gas very similar to those obtained in the $G^{0} W^{0}$ approximation, due to a large cancel- lation of vertex corrections. Nonetheless, due to the wellknown differences between the dynamical response of the noble metals and the homogeneous electron gas, the impact of exchange and correlation on the relaxation of hot electrons in these materials required further investigation.

Our first-principles calculations start by solving selfconsistently the Kohn-Sham equations of density functional theory (DFT), ${ }^{25,26}$ within the local density approximation (LDA) and with the use of a plane-wave expansion of the single-particle Kohn-Sham Bloch states. The electron-ion interaction is described by a nonlocal, norm-conserving ionic pseudopotential, ${ }^{27}$ by keeping all $d$ electrons as valence electrons. The single-particle Bloch states are then used to compute the $G W \Gamma$ electron self-energy and hot-electron inelastic lifetimes. For comparison, we also compute $G^{0} W^{0}, G^{0} W$, and $G W^{0} \Gamma$ decay rates, with no inclusion of XC effects, with inclusion of XC effects beyond the RPA in the screened interaction $W$, and with inclusion of $\mathrm{XC}$ effects beyond the $G^{0} W^{0}$ in the expansion of the electron self-energy in terms of the RPA screened interaction $W^{0}$, respectively. Our results indicate that the overall effect of short-range $\mathrm{XC}$ is small and $G W \Gamma$ linewidths are close to their $G^{0} W^{0}$ counterparts, as occurs in the case of a FEG.

In order to establish the role that occupied $d$ states play in the relaxation of hot electrons in the noble metals, we also use a pseudopotential with all $d$ electrons assigned to the core. We find that a major contribution from occupied $d$ states participating in the screening of $e-e$ interactions yields lifetimes of low-energy excited hot electrons that are larger than in the absence of $d$ states by a factor of $\sim 2$ in $\mathrm{Cu}$ and by a factor of $\sim 2.5$ in $\mathrm{Ag}$ and $\mathrm{Au}$.

This paper is organized as follows. Explicit expressions for the electron decay rate (inelastic lifetime broadening) in a FEG and periodic crystals are derived in Sec. II, in the $G W \Gamma$ approximation of many-body theory. The results of numerical calculations of hot-electron lifetimes in the noble metals $\mathrm{Cu}, \mathrm{Ag}$, and $\mathrm{Au}$ are presented in Sec. III. The summary and conclusions are given in Sec. IV. Unless stated otherwise, atomic units are used throughout, i.e., $e^{2}=\hbar=m_{e}=1$. 


\section{THEORY}

Let us consider an arbitrary many-electron system of density $n_{0}(\mathbf{r})$. In the framework of many-body theory, the damping rate or reciprocal lifetime of a quasiparticle in the singleparticle state $\phi_{i}(\mathbf{r})$ of energy $\varepsilon_{i}\left(\varepsilon_{i}>\varepsilon_{F}, \varepsilon_{F}\right.$ being the Fermi energy) is obtained as the projection of the imaginary part of the electron self-energy $\Sigma\left(\mathbf{r}, \mathbf{r}^{\prime} ; \varepsilon_{i}\right)$ over the quasiparticlestate itself ${ }^{3}$

$$
\tau_{i}^{-1}=-2 \int d \mathbf{r} \int d \mathbf{r}^{\prime} \phi_{i}^{*}(\mathbf{r}) \operatorname{Im} \Sigma\left(\mathbf{r}, \mathbf{r}^{\prime} ; \varepsilon_{i}\right) \phi_{i}\left(\mathbf{r}^{\prime}\right)
$$

Within many-body perturbation theory, it is possible to obtain the electron self-energy as a series in the bare Coulomb interaction $v\left(\mathbf{r}, \mathbf{r}^{\prime}\right)$, but due to the long range of this interaction such a perturbation series contains divergent contributions. Therefore, the electron self-energy is usually rewritten as a series in the frequency-dependent screened interaction $W\left(\mathbf{r}, \mathbf{r}^{\prime} ; \omega\right)$. To lowest order in the screened interaction, the self-energy is obtained by integrating the product of the interacting Green function $G\left(\mathbf{r}, \mathbf{r}^{\prime}, \varepsilon_{i}-\omega\right)$ and the screened interaction $W\left(\mathbf{r}, \mathbf{r}^{\prime} ; \omega\right)$, and is therefore called the $G W$ self-energy. If one further replaces the interacting Green function by its noninteracting counterpart $G^{0}\left(\mathbf{r}, \mathbf{r}^{\prime}, \varepsilon_{i}\right.$ $-\omega$ ), one finds the $G^{0} W$ self-energy and from Eq. (1) the following expression for the $G^{0} W$ lifetime broadening:

$$
\begin{aligned}
\tau_{i}^{-1}= & -2 \sum_{f} \int d \mathbf{r} \int d \mathbf{r}^{\prime} \phi_{i}^{*}(\mathbf{r}) \phi_{f}^{*}\left(\mathbf{r}^{\prime}\right) \\
& \times \operatorname{Im} W\left(\mathbf{r}, \mathbf{r}^{\prime} ; \varepsilon_{i}-\varepsilon_{f}\right) \phi_{i}\left(\mathbf{r}^{\prime}\right) \phi_{f}(\mathbf{r}),
\end{aligned}
$$

where the sum is extended over a complete set of singleparticle states $\phi_{f}(\mathbf{r})$ of energy $\varepsilon_{f}\left(\varepsilon_{F} \leqslant \varepsilon_{f} \leqslant \varepsilon_{i}\right)$. The screened interaction $W\left(\mathbf{r}, \mathbf{r}^{\prime} ; \omega\right)$ can be rigorously expressed as follows:

$$
\begin{aligned}
W\left(\mathbf{r}, \mathbf{r}^{\prime} ; \omega\right)= & v\left(\mathbf{r}, \mathbf{r}^{\prime}\right)+\int d \mathbf{r}_{1} \int d \mathbf{r}_{2} v\left(\mathbf{r}, \mathbf{r}_{1}\right) \\
& \times \chi\left(\mathbf{r}_{1}, \mathbf{r}_{2} ; \omega\right) v\left(\mathbf{r}_{2}, \mathbf{r}^{\prime}\right),
\end{aligned}
$$

$\chi(\mathbf{r}, \mathbf{r} ; \omega)$ being the time-ordered density-response function of the many-electron system, which for the positive frequencies $(\omega>0)$ entering Eq. (2) coincides with the retarded density-response function of linear-response theory. In the framework of time-dependent DFT (TDDFT), ${ }^{28}$ the exact retarded density-response function is obtained by solving the following integral equation: ${ }^{29}$

$$
\begin{aligned}
\chi\left(\mathbf{r}, \mathbf{r}^{\prime} ; \omega\right)= & \chi^{0}\left(\mathbf{r}, \mathbf{r}^{\prime} ; \omega\right)+\int d \mathbf{r}_{1} \int d \mathbf{r}_{2} \chi^{0}\left(\mathbf{r}, \mathbf{r}_{1} ; \omega\right) \\
& \times\left\{v\left(\mathbf{r}_{1}, \mathbf{r}_{2}\right)+f^{\mathrm{XC}}\left[n_{0}\right]\left(\mathbf{r}_{1}, \mathbf{r}_{2} ; \omega\right)\right\} \chi\left(\mathbf{r}_{2}, \mathbf{r}^{\prime} ; \omega\right),
\end{aligned}
$$

where $\chi^{0}\left(\mathbf{r}, \mathbf{r}^{\prime} ; \omega\right)$ denotes the density-response function of noninteracting electrons

$$
\chi^{0}\left(\mathbf{r}, \mathbf{r}^{\prime} ; \omega\right)=2 \sum_{i, j} \frac{f_{i}-f_{j}}{\varepsilon_{i}-\varepsilon_{j}+\omega+i \eta} \phi_{i}(\mathbf{r}) \phi_{j}^{*}(\mathbf{r}) \phi_{j}\left(\mathbf{r}^{\prime}\right) \phi_{i}^{*}\left(\mathbf{r}^{\prime}\right)
$$

Here, $\phi_{i}(\mathbf{r})$ and $\varepsilon_{i}$ denote the eigenfunctions and eigenvalues of the Kohn-Sham Hamiltonian of DFT, $f_{i}$ are Fermi-Dirac occupation factors, $\eta$ is a positive infinitesimal, and the frequency-dependent XC kernel $f^{\mathrm{XC}}\left[n_{0}\right]\left(\mathbf{r}, \mathbf{r}^{\prime} ; \omega\right)$ is the functional derivative of the frequency-dependent $\mathrm{XC}$ potential $V_{\mathrm{XC}}[n](\mathbf{r}, \omega)$ of TDDFT, to be evaluated at $n_{0}(\mathbf{r})$ :

$$
f^{\mathrm{XC}}\left[n_{0}\right]\left(\mathbf{r}, \mathbf{r}^{\prime} ; \omega\right)=\left.\frac{\delta V_{\mathrm{XC}}[n](\mathbf{r}, \omega)}{\delta n\left(\mathbf{r}^{\prime}, \omega\right)}\right|_{n=n_{0}} .
$$

In the RPA, $f^{\mathrm{XC}}\left[n_{0}\right]\left(\mathbf{r}, \mathbf{r}^{\prime} ; \omega\right)$ is set equal to zero and Eq. (2) yields the so-called $G^{0} W^{0}$ lifetime broadening. ${ }^{35}$ In the adiabatic LDA (ALDA), ${ }^{36}$

$$
f^{\mathrm{XC}}\left(\mathbf{r}, \mathbf{r}^{\prime} ; \omega\right)=\left.\frac{d V_{\mathrm{XC}}(n)}{d n}\right|_{n=n_{0}(\mathbf{r})} \delta\left(\mathbf{r}-\mathbf{r}^{\prime}\right),
$$

$V_{\mathrm{XC}}(n)$ being the static $\mathrm{XC}$ potential of a uniform electron gas of density $n$.

The XC kernel $f^{\mathrm{XC}}\left[n_{0}\right]\left(\mathbf{r}, \mathbf{r}^{\prime} ; \omega\right)$, which is absent in the RPA, accounts for the presence of an XC hole associated to all electrons in the Fermi sea. Hence, one might be tempted to conclude that the full $G^{0} W$ approximation [with the formally exact screened interaction $W$ of Eq. (3)] should be a better approximation than its $G^{0} W^{0}$ counterpart (with the screened interaction $W$ evaluated in the RPA). However, the $\mathrm{XC}$ hole associated to the excited hot electron is still absent in the $G^{0} W$ approximation. Therefore, if one goes beyond RPA in the description of $W$, one should also go beyond the $G^{0} W$ approximation in the expansion of the electron selfenergy in powers of $W$. By including XC effects both beyond RPA in the description of $W$ and beyond $G^{0} W$ in the description of the self-energy, ${ }^{23,24}$ the so-called $G W \Gamma$ approximation yields a lifetime broadening that is of the $G^{0} W$ form [see Eq. (2)], but with the actual screened interaction $W\left(\mathbf{r}, \mathbf{r}^{\prime} ; \omega\right)$ replaced by a new effective screened interaction

$$
\begin{aligned}
\tilde{W}\left(\mathbf{r}, \mathbf{r}^{\prime} ; \omega\right)= & v\left(\mathbf{r}, \mathbf{r}^{\prime}\right)+\int d \mathbf{r}_{1} \int d \mathbf{r}_{2}\left\{v\left(\mathbf{r}, \mathbf{r}_{1}\right)\right. \\
& \left.+f^{\mathrm{XC}}\left[n_{0}\right]\left(\mathbf{r}, \mathbf{r}_{1} ; \omega\right)\right\}\left(\mathbf{r}_{1}, \mathbf{r}_{2} ; \omega\right) v\left(\mathbf{r}_{2}, \mathbf{r}^{\prime}\right),
\end{aligned}
$$

which includes all powers in $W$ beyond the $G^{0} W$ approximation.

\section{A. Free-electron gas}

In the case of a uniform FEG, there is translational invariance in all directions, the single-particle states entering Eqs. (2) and (5) are momentum eigenfunctions $\phi_{\mathbf{k}}(\mathbf{r})=\exp (i \mathbf{k} \cdot \mathbf{r})$ of energy $\varepsilon_{\mathbf{k}}=k^{2} / 2$, and Eq. (2) is easily found to yield

$$
\tau_{\mathbf{k}}^{-1}=-2 \int \frac{d \mathbf{q}}{(2 \pi)^{3}} \operatorname{Im} W\left(\mathbf{q}, \varepsilon_{\mathbf{k}}-\varepsilon_{\mathbf{k}-\mathbf{q}}\right),
$$

the integral being subject to the condition that $\varepsilon_{F}<\varepsilon_{\mathbf{k}-\mathbf{q}}<\varepsilon_{\mathbf{k}}$, and $W(\mathbf{q}, \omega)$ being the Fourier transform of the screened in- 
teraction $W\left(\mathbf{r}, \mathbf{r}^{\prime} ; \omega\right)$ of Eq. (3), which in the $G W \Gamma$ approximation should be replaced by the effective screened interaction $\widetilde{W}\left(\mathbf{r}, \mathbf{r}^{\prime} ; \omega\right)$ of Eq. (8). We note that Eq. (9) with $W(\mathbf{q}, \omega)$ replaced by the Fourier transform of $\widetilde{W}\left(\mathbf{r}, \mathbf{r}^{\prime} ; \omega\right)$ yields precisely the decay rate that one would obtain from the $G W \Gamma$ self-energy of Ref. 23.

\section{B. Periodic crystals}

For periodic crystals, the single-particle states entering Eq. (2) are Bloch states $\phi_{\mathbf{k}, i}(\mathbf{r})$ and $\phi_{\mathbf{k}-\mathbf{q}, f}(\mathbf{r})$ with energies $\varepsilon_{\mathbf{k}, i}$ and $\varepsilon_{\mathbf{k}-\mathbf{q}, f}, i$ and $f$ denoting band indices. Hence, Eq. (2) yields

$$
\begin{aligned}
\tau_{\mathbf{k}, i}^{-1}= & \frac{1}{\pi^{2}} \sum_{f} \int_{\mathrm{BZ}} d \mathbf{q} \sum_{\mathbf{G}, \mathbf{G}^{\prime}} B_{\mathbf{k}, i ; \mathbf{k}-\mathbf{q}, f}^{*}(\mathbf{G}) B_{\mathbf{k}, i ; \mathbf{k}-\mathbf{q}, f}\left(\mathbf{G}^{\prime}\right) \\
& \times \operatorname{Im} W_{\mathbf{G}, \mathbf{G}^{\prime}}\left(\mathbf{q}, \varepsilon_{\mathbf{k}, i}-\varepsilon_{\mathbf{k}-\mathbf{q}, f}\right),
\end{aligned}
$$

where the integral is extended over the first Brillouin Zone (BZ), the vectors $\mathbf{G}$ and $\mathbf{G}^{\prime}$ are reciprocal-lattice vectors,

$$
B_{\mathbf{k}, i ; \mathbf{k}-\mathbf{q}, f}(\mathbf{G})=\int d \mathbf{r} \phi_{\mathbf{k}, i}^{*}(\mathbf{r}) e^{i(\mathbf{q}+\mathbf{G}) \cdot \mathbf{r}} \phi_{\mathbf{k}-\mathbf{q}, f}(\mathbf{r}),
$$

and $W_{\mathbf{G}, \mathbf{G}^{\prime}}(\mathbf{q}, \omega)$ denote the Fourier coefficients of the screened interaction $W\left(\mathbf{r}, \mathbf{r}^{\prime} ; \omega\right)$, which are usually expressed as

$$
W_{\mathbf{G}, \mathbf{G}^{\prime}}(\mathbf{q}, \omega)=\epsilon_{\mathbf{G}, \mathbf{G}^{\prime}}^{-1}(\mathbf{q}, \omega) v_{\mathbf{G}^{\prime}}(\mathbf{q}),
$$

$v_{\mathbf{G}}(\mathbf{q})=4 \pi /|\mathbf{q}+\mathbf{G}|^{2}$ being the Fourier transform of the bare Coulomb interaction, and $\epsilon_{\mathbf{G}, \mathbf{G}^{\prime}}^{-1}(\mathbf{q}, \omega)$ being the so-called inverse dielectric matrix.

We remark that for a given hot-electron energy $\varepsilon$ there are in general various possible wave vectors and bands. Since $\tau_{S \mathbf{k}, i}^{-1}=\tau_{\mathbf{k}, i}^{-1}, S$ denoting a point-group symmetry operation in the crystal, one only needs to consider states inside the irreducible wedge of the BZ (IBZ). An energy-dependent reciprocal lifetime $\tau^{-1}(\varepsilon)$ can then be defined by doing a weighed average over all wave vectors and bands lying with the same energy in the IBZ.

\section{1. $G^{0} W^{0}$ approximation}

In the $G^{0} W^{0}$ approximation, the XC kernel $f^{\mathrm{XC}}\left[n_{0}\right]\left(\mathbf{r}, \mathbf{r}^{\prime} ; \omega\right)$ entering Eqs. (4) and (8) is set equal to zero. Hence, in this approximation the dielectric matrix is

$$
\epsilon_{\mathbf{G}, \mathbf{G}^{\prime}}(\mathbf{q}, \omega)=\delta_{\mathbf{G}, \mathbf{G}^{\prime}}-v_{\mathbf{G}}(\mathbf{q}) \chi_{\mathbf{G}, \mathbf{G}^{\prime}}^{0},(\mathbf{q}, \omega),
$$

$\chi_{\mathbf{G}, \mathbf{G}^{\prime}}^{0},(\mathbf{q}, \omega)$ being the Fourier coefficients of the noninteracting density-response function of Eq. (5):

$$
\begin{aligned}
\chi_{\mathbf{G}, \mathbf{G}^{\prime}}^{0}(\mathbf{q}, \omega)= & 2 \int_{\mathrm{BZ}} \frac{d \mathbf{k}}{(2 \pi)^{3}} \sum_{n, n^{\prime}} \frac{f_{\mathbf{k}, n}-f_{\mathbf{k}+\mathbf{q}, n^{\prime}}}{\epsilon_{\mathbf{k}, n}-\epsilon_{\mathbf{k}+\mathbf{q}, n^{\prime}}+\omega+i \eta} \\
& \times\left\langle\phi_{\mathbf{k}, n}\left|e^{-i(\mathbf{q}+\mathbf{G}) \cdot \mathbf{r}}\right| \phi_{\mathbf{k}+\mathbf{q}, n^{\prime}}\right\rangle \\
& \times\left\langle\phi_{\mathbf{k}+\mathbf{q}, n^{\prime}}\left|e^{i\left(\mathbf{q}+\mathbf{G}^{\prime}\right) \cdot \mathbf{r}}\right| \phi_{\mathbf{k}, n}\right\rangle .
\end{aligned}
$$

Couplings of the wave vector $\mathbf{q}+\mathbf{G}$ to wave vectors $\mathbf{q}$ $+\mathbf{G}^{\prime}$ with $\mathbf{G} \neq \mathbf{G}^{\prime}$ appear as a consequence of the existence of electron-density variations in the solid. If these terms, representing the so-called crystalline local-field effects (LFE), are neglected, one can write Eq. (10) as

$$
\begin{aligned}
\tau_{\mathbf{k}, i}^{-1}= & \frac{1}{\pi^{2}} \sum_{f} \int_{\mathrm{BZ}} d \mathbf{q} \sum_{\mathbf{G}} \frac{\left|B_{\mathbf{k}, i ; \mathbf{k}-\mathbf{q}, f}(\mathbf{G})\right|^{2}}{|\mathbf{q}+\mathbf{G}|^{2}} \\
& \times \frac{\operatorname{Im}\left[\epsilon_{\mathbf{G}, \mathbf{G}}\left(\mathbf{q}, \varepsilon_{\mathbf{k}, i}-\varepsilon_{\mathbf{k}-\mathbf{q}, f}\right)\right]}{\left|\epsilon_{\mathbf{G}, \mathbf{G}}\left(\mathbf{q}, \varepsilon_{\mathbf{k}, i}-\varepsilon_{\mathbf{k}-\mathbf{q}, f}\right)\right|^{2}} .
\end{aligned}
$$

This expression accounts explicitly for the three main ingredients entering the hot-electron decay process. First of all, the coupling of the hot electron with available states above the Fermi level is dictated by the matrix elements $B_{\mathbf{k}, i ; \mathbf{k}-\mathbf{q}, f}(\mathbf{G})$. Secondly, the imaginary part of the dielectric matrix $\epsilon_{\mathbf{G}, \mathbf{G}}\left(\mathbf{q}, \varepsilon_{\mathbf{k}, i}-\varepsilon_{\mathbf{k}-\mathbf{q}, f}\right)$ represents a measure of the number of states available for the creation of $e-h$ pairs with momentum and energy $\mathbf{q}+\mathbf{G}$ and $\varepsilon_{\mathbf{k}, i}-\varepsilon_{\mathbf{k}-\mathbf{q}, f}$, respectively. Thirdly, the dielectric matrix in the denominator accounts for the many-body $e-e$ interactions in the Fermi sea, which dynamically screen the interaction with the external hot electron.

\section{2. $G^{0} W$ approximation}

In the $G^{0} W$ approximation, the Fourier coefficients $W_{\mathbf{G}, \mathbf{G}^{\prime}}(\mathbf{q}, \omega)$ still take the form of Eq. (12), but with the inverse dielectric matrix now given by the following general expression:

$$
\epsilon_{\mathbf{G}, \mathbf{G}^{\prime}}^{-1}(\mathbf{q}, \omega)=\delta_{\mathbf{G}, \mathbf{G}^{\prime}}+v_{\mathbf{G}}(\mathbf{q}) \chi_{\mathbf{G}, \mathbf{G}^{\prime}}(\mathbf{q}, \omega),
$$

where $\chi_{\mathbf{G}, \mathbf{G}^{\prime}}(\mathbf{q}, \omega)$ are the Fourier coefficients of the interacting density-response function of Eq. (4):

$$
\begin{aligned}
\chi_{\mathbf{G}, \mathbf{G}^{\prime}}(\mathbf{q}, \omega)= & \chi_{\mathbf{G}_{\mathbf{G}, \mathbf{G}^{\prime}}}^{0}(\mathbf{q}, \omega)+\sum_{\mathbf{G}_{1}, \mathbf{G}_{2}} \chi_{\mathbf{G}, \mathbf{G}_{1}}^{0}(\mathbf{q}, \omega) \\
& \times\left\{v_{\mathbf{G}_{1}}(\mathbf{q})+f_{\mathbf{G}_{1}, \mathbf{G}_{2}}^{\mathrm{XC}}\left[n_{0}\right](\mathbf{q}, \omega)\right\} \chi_{\mathbf{G}_{2}, \mathbf{G}^{\prime}}(\mathbf{q}, \omega),
\end{aligned}
$$

$f_{\mathbf{G}, \mathbf{G}^{\prime}}^{\mathrm{XC}}\left[n_{0}\right](\mathbf{q}, \omega)$ being the Fourier coefficients of the XC kernel of Eq. (6). In the ALDA,

$$
f_{\mathbf{G}, \mathbf{G}^{\prime}}^{\mathrm{XC}}(\mathbf{q}, \omega)=\left.\int d \mathbf{r} \mathrm{e}^{-i\left(\mathbf{G}-\mathbf{G}^{\prime}\right) \cdot \mathbf{r}} \frac{d V_{\mathrm{xc}}(n)}{d n}\right|_{n=n_{0}(\mathbf{r})} .
$$

\section{GW approximation}

In the $G W \Gamma$ approximation, the lifetime broadening is still of the form of Eqs. (10)-(12), but with the test-charge-testcharge inverse dielectric matrix of Eq. (16) replaced by the new test-charge-electron inverse dielectric matrix

$$
\begin{aligned}
\tilde{\boldsymbol{\epsilon}}_{\mathbf{G}, \mathbf{G}^{\prime}}^{-1}(\mathbf{q}, \omega)= & \delta_{\mathbf{G}, \mathbf{G}^{\prime}}+\sum_{\mathbf{G}^{\prime \prime}}\left\{v_{\mathbf{G}}(\mathbf{q}) \delta_{\mathbf{G}, \mathbf{G}^{\prime \prime}}\right. \\
& \left.+f_{\mathbf{G}, \mathbf{G}^{\prime \prime}}^{\mathrm{XC}}\left[n_{0}\right](\mathbf{q}, \omega)\right\} \chi_{\mathbf{G}^{\prime \prime}, \mathbf{G}^{\prime}}(\mathbf{q}, \omega),
\end{aligned}
$$

where $\chi_{\mathbf{G}, \mathbf{G}^{\prime}}(\mathbf{q}, \omega)$ are the Fourier coefficients of Eq. (17). If 
one sets the XC kernel $f_{\mathbf{G}_{\mathbf{G}} \mathbf{G}^{\prime}}^{\mathrm{XC}}\left[n_{0}\right](\mathbf{q}, \omega)$ entering Eqs. (17) and (19) equal to zero, one finds the $G^{0} W^{0}$ approximation. Instead, if one only sets the XC kernel $f_{\mathbf{G}, \mathbf{G}}^{\mathrm{XC}},\left[n_{0}\right](\mathbf{q}, \omega)$ entering Eq. (17) equal to zero, one finds the $G W^{0} \Gamma$ approximation.

\section{RESULTS AND DISCUSSION}

In the calculations presented in this section, all the singleparticle Bloch states and energies entering Eqs. (10), (11), and (14) are taken to be the eigenfunctions and eigenvalues of the LDA Kohn-Sham Hamiltonian of DFT. We expand the single-particle Bloch states in a plane-wave basis, we invoke the LDA with the Perdew-Zunger parametrization ${ }^{30}$ of the quantum Monte Carlo uniform-gas XC energy of Ceperly and Alder, ${ }^{31}$ and we describe the electron-ion interaction with the use of a nonlocal, norm-conserving ionic pseudopotential. ${ }^{27}$

$\mathrm{Cu}, \mathrm{Ag}$, and $\mathrm{Au}$ are noble metals with entirely filled $n d$ bands, $n$ being 3, 4, and 5, respectively. Slightly below the Fermi level (at $\varepsilon-\varepsilon_{F} \sim 2 \mathrm{eV}$ in $\mathrm{Cu}$ and $\mathrm{Au}$, and at $\varepsilon-\varepsilon_{F}$ $\sim 4 \mathrm{eV}$ in $\mathrm{Ag}$ ) there are $d$ bands capable of holding 10 electrons per atom. The one remaining $(n+1) s$ electron per atom occupies a free-electron-like band below and above the $d$ bands. Hence, a combined description of the localized $n d^{10}$ and delocalized $(n+1) s^{1}$ electrons is needed in order to address the actual electronic response of these materials.

The results presented below have been found by either keeping all $n d^{10}$ and $(n+1) s^{1}$ electrons (full calculation) or keeping only the $(n+1) s^{1}$ electrons ( $s$ calculation) as valence electrons in the generation of the pseudopotential. The full calculation has required a kinetic-energy cutoff as large as $75 \mathrm{Ry} .{ }^{37}$ Well-converged results have been found for all hotelectron energies under study $\left(0.5 \mathrm{eV} \leqslant \varepsilon-\varepsilon_{F} \leqslant 3.5 \mathrm{eV}\right)$, with the inclusion of conduction bands up to a maximum energy of $\sim 25 \mathrm{eV}$ above the Fermi level. Samplings of the BZ have been performed on a $20 \times 20 \times 20$ mesh containing 256 points in the IBZ, although well-converged results have sometimes been obtained on $16 \times 16 \times 16$ meshes. Reciprocal-space sums have been extended over $15 \mathrm{G}$ vectors, the magnitude of the maximum momentum transfer $\mathbf{q}$ $+\mathbf{G}$ being well over the upper limit of $\sim 2 q_{F}, q_{F}$ being the Fermi momentum.

\section{A. Copper}

Figure 1 shows our full first-principles $G W \Gamma$ calculation of the average lifetime $\tau(E)$ of hot electrons in $\mathrm{Cu}$ (solid circles), as obtained in the ALDA from Eqs. (10)-(12) but with the inverse dielectric matrix $\epsilon_{\mathbf{G}, \mathbf{G}^{\prime}}^{-1}(\mathbf{q}, \omega)$ replaced by that of Eq. (19). For comparison, we also plot in the same figure our full first-principles $G^{0} W^{0}$ calculation (open circles), which reproduces previous calculations, ${ }^{13}$ firstprinciples $G W \Gamma$ and $G^{0} W^{0} s$ calculations (solid and open squares), and $G W \Gamma$ and $G^{0} W^{0}$ FEG calculations with the electron density $n_{0}$ equal to that of $4 s^{1}$ electrons in $\mathrm{Cu}$ (solid and dashed lines).

We note from Fig. 1 that XC effects yield hot-electron lifetimes that are larger than in the absence of exchange and correlation by no more than $\sim 3 \%$ in the whole hot-electron

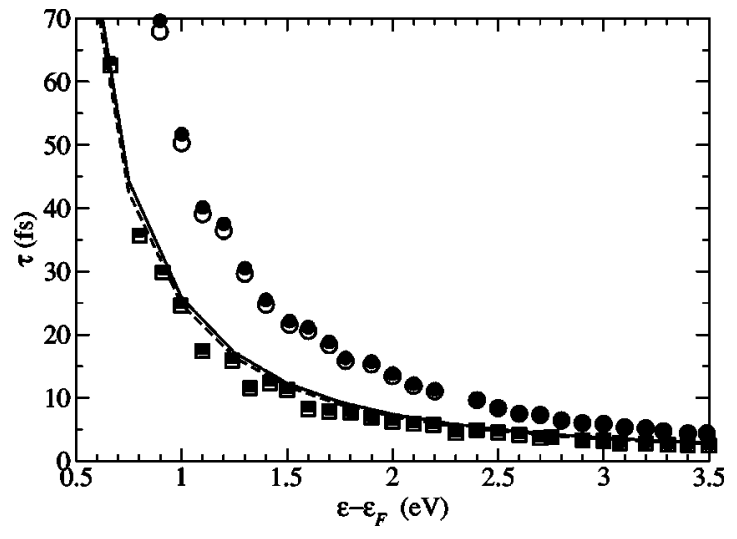

FIG. 1. Average lifetime of hot electrons in $\mathrm{Cu}$, as a function of the hot-electron energy $\varepsilon-\varepsilon_{F}$ with respect to the Fermi level. Solid and open circles represent full $G W \Gamma$ and $G^{0} W^{0}$ calculations, as obtained from Eqs. (10)-(12) with the test-charge-electron inverse dielectric matrix of Eq. (19) and with the RPA dielectric matrix of Eq. (13), respectively. Solid and open squares represent the corresponding $G W \Gamma$ and $G^{0} W^{0} s$ calculations, respectively, where the $3 d$ shell is assigned to the core in the generation of the pseudopotential. Solid and dashed lines represent $G W \Gamma$ and $G^{0} W^{0}$ FEG calculations, as obtained from Eq. (9) with the electron-density parameter $r_{s}$ $=2.67\left[r_{s}=\left(3 / 4 \pi n_{0}\right)^{1 / 3}\right]$ corresponding to the average density $n_{0}$ of $4 s^{1}$ electrons in $\mathrm{Cu}$. The Fourier coefficients $f_{\mathbf{G}, \mathbf{G}^{\prime}}^{\mathrm{XC}},\left[n_{0}\right](\mathbf{q}, \omega)$ and $f^{\mathrm{XC}}\left[n_{0}\right](\mathbf{q}, \omega)$ entering the crystal and FEG calculations have both been calculated in the ALDA with use of the Perdew-Zunger XC potential of a uniform electron gas.

energy range under study, as occurs when $d$ electrons are assigned to the core ( $s$ calculations) and in the case of a FEG. Exchange-correlation effects included in the $G W \Gamma$ scheme have two sources, as discussed in Sec. II. First, there is the reduction of the screening due to the presence of an XC hole associated to occupied states below the Fermi level, as in the $G^{0} W$ approximation. Secondly, there is the XC hole associated to the excited hot electron over the Fermi level, as in the $G W^{0} \Gamma$ approximation. These contributions have opposite signs and it is the latter which dominates.

The impact of XC effects on the lifetime of hot electrons in $\mathrm{Cu}$ is illustrated in Fig. 2, where $G^{0} W, G W^{0} \Gamma$, and $G W \Gamma$ calculations are compared to their $G^{0} W^{0}$ counterparts, with (upper panel) and without (lower panel) inclusion of $d$ states. The impact of exchange and correlation on either screening or the bare interaction of the hot electron with the Fermi sea, which is significant in the absence of $d$ states (lower panel), is considerably reduced in the presence of $d$ electrons. Moreover, these small XC effects partially compensate each other, leading to an overall effect of no more than $3 \%$.

Now we focus on the role that localized $d$ bands play in the decay mechanism of hot electrons in $\mathrm{Cu}$. This issue has been investigated before, ${ }^{13}$ by replacing the various contributions to Eq. (15) by the corresponding FEG contributions. Here, we follow a more meaningful procedure. As in Ref. 13 we use Eq. (15) (hence, with inclusion of neither XC effects nor crystalline local-field corrections), but now we replace the various contributions to the full calculation by the corresponding $s$ contributions where the $3 d$ shell is assigned to the core. These contributions are (i) the imaginary part of the 

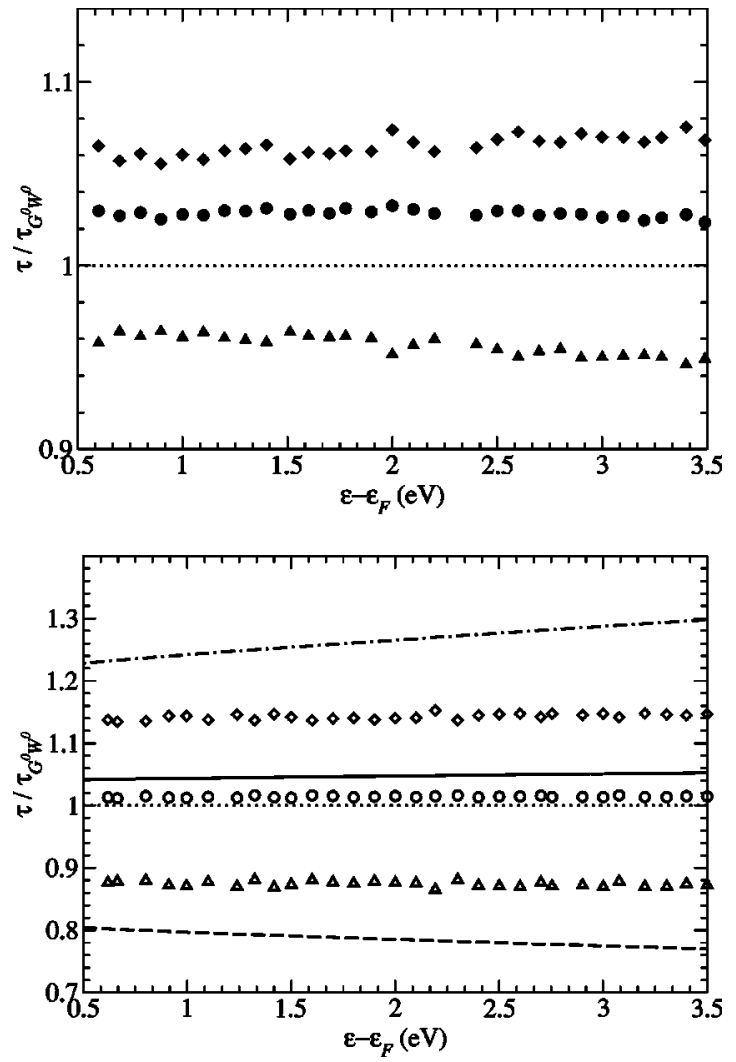

FIG. 2. Impact of exchange and correlation on the lifetime of hot electrons in $\mathrm{Cu}$, as a function of the hot-electron energy $\varepsilon-\varepsilon_{F}$ with respect to the Fermi level. Triangles, diamonds, and circles represent the ratios $\tau_{G^{0} W} / \tau_{G^{0} W^{0}}, \tau_{G W^{0} \Gamma} / \tau_{G^{0} W^{0}}$, and $\tau_{G W \Gamma} / \tau_{G^{0} W^{0}}$ in the presence (upper panel) and in the absence (lower panel) of $3 d^{10}$ occupied states, respectively. Dashed, dashed-dotted and solid lines represent the corresponding ratios for a FEG with $r_{s}=2.67$.

dielectric matrix $\epsilon_{\mathbf{G}, \mathbf{G}}(\mathbf{q}, \omega)$, which represents a measure of both the number of states available for the creation of $e-h$ pairs and the coupling between states below and above the Fermi level, (ii) the denominator $\left|\epsilon_{\mathbf{G}, \mathbf{G}}(\mathbf{q}, \omega)\right|^{2}$, which accounts for screening effects, and (iii) the matrix elements $B_{\mathbf{k}, i ; \mathbf{k}-\mathbf{q}, f}(\mathbf{G})$ accounting for the coupling of the hot electron with available states above the Fermi level.

Figure 3 shows first-principles $G^{0} W^{0}$ calculations of the scaled lifetime $\tau(\varepsilon) \times\left(\varepsilon-\varepsilon_{F}\right)^{2}$ of hot electrons in $\mathrm{Cu}$, as obtained from Eq. (15). We start with the $s$ calculation (open squares), which we obtain by assigning the $3 d$ shell to the core in the generation of the pseudopotential. This calculation nearly coincides with the $G^{0} W^{0}$ lifetime broadening of hot electrons in a free-gas of $4 s^{1}$ electrons (dashed line), which must be a consequence of the fact that band-structure effects are almost entirely due to the presence of occupied $d$ bands. In $\mathrm{Cu}$, localized $d$ bands contribute to the decay of $s p$ hot electrons by either opening a $d$-band scattering channel at $\sim 2 \mathrm{eV}$ or by screening the $e-e$ interactions. While the opening of the $d$-band scattering channel modifies the imaginary part of the dielectric matrix $\epsilon_{\mathbf{G}, \mathbf{G}}(\mathbf{q}, \omega)$, the screening of $d$ electrons modifies the denominator $\left|\boldsymbol{\epsilon}_{\mathbf{G}, \mathbf{G}}(\mathbf{q}, \omega)\right|^{2}$. Hence, we have gone beyond the $s$ calculation (open squares) by first including all $d$ states only in the evaluation of $\operatorname{Im}\left[\epsilon_{\mathbf{G}, \mathbf{G}}(\mathbf{q}, \omega)\right]$

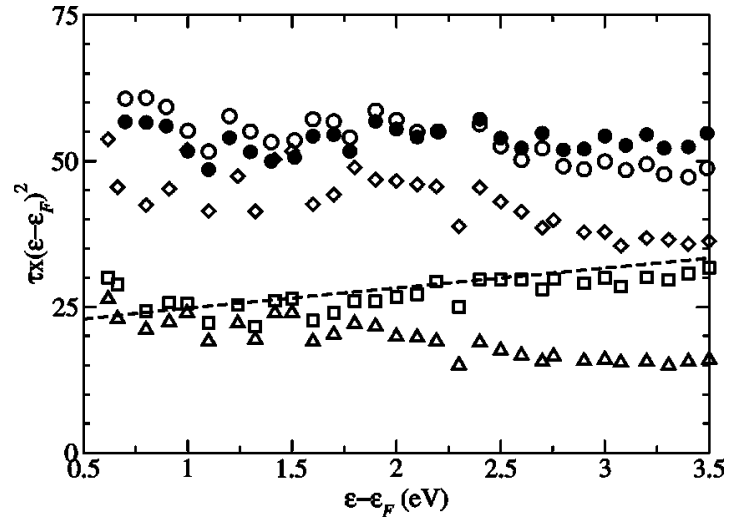

FIG. 3. Scaled lifetimes $\tau(\varepsilon) \times\left(\varepsilon-\varepsilon_{F}\right)^{2}$ of hot electrons in $\mathrm{Cu}$. Open circles, squares, triangles, and diamonds represent $G^{0} W^{0}$ calculations obtained from Eq. (15) (hence, with no inclusion of crystalline local-field effects), (i) in the presence of $3 d$ states (open circles), (ii) in the absence of $3 d$ states (squares), (iii) including $3 d$ states only in the evaluation of the imaginary part of $\epsilon_{\mathbf{G}, \mathbf{G}}(\mathbf{q}, \omega)$ (triangles), and (iv) including $3 d$ states in the evaluation of the imaginary part of $\epsilon_{\mathbf{G}, \mathbf{G}}(\mathbf{q}, \omega)$ as well as in the evaluation of the denominator $\left|\epsilon_{\mathbf{G}, \mathbf{G}}(\mathbf{q}, \omega)\right|^{2}$ (diamonds). Solid circles represent the full $G W \Gamma$ matricial calculation of Fig. 1, but now multiplied by $\left(\varepsilon-\varepsilon_{F}\right)^{2}$. The dashed line represents the $G^{0} W^{0}$ calculation for hot electrons in a FEG with $r_{s}=2.67$.

(triangles) and then including all $d$ states also in the evaluation of $\left|\epsilon_{\mathbf{G}, \mathbf{G}}(\mathbf{q}, \omega)\right|^{-2}$ (diamonds). These calculations clearly indicate that: (i) occupied $d$ bands yield an slightly enhanced hot-electron decay (reduced lifetime) at the opening of the $d$-band scattering channel $\left(\varepsilon-\varepsilon_{F}>2 \mathrm{eV}\right)$, which is considerably smaller than expected from the greatly enhanced density of states at these energies due to the small coupling between $d$ states below and $s p$ states above the Fermi level for the creation of $e-h$ pairs and (ii) the key role that $d$ electrons play in the hot-electron decay is mainly due to screening effects. We also note that the full diagonal $G^{0} W^{0}$ calculation (open circles), which includes neither crystalline local-field corrections nor XC effects, is very close to the full $G W \Gamma$ calculation (solid circles). This shows that the overall impact of these effects in this material is never larger than a few per cent.

The screening of $d$ electrons in the noble metals was included by Quinn, ${ }^{32}$ by embedding the $n s^{1}$ free electrons in a polarizable background of $d$ electrons characterized by a dielectric constant $\epsilon_{d}$ instead of unity. The corrected lifetime is then found to be larger than in the absence of $d$ electrons by roughly a factor of $\epsilon_{d}^{1 / 2}$. For $\mathrm{Cu}$ and $\mathrm{Au} \epsilon_{d}=5.6$ and for $\mathrm{Ag}$ $\epsilon_{d}=3.4 .{ }^{33}$ Hence, this simple model yields corrected lifetimes in $\mathrm{Cu}$ that are larger than in a FEG by roughly a factor of $\sim 2.5$, in qualitative agreement with first-principles calculations. Nevertheless, this model cannot account for the existing differences between the impact of occupied $d$ states in the lifetime broadening of hot electrons in $\mathrm{Cu}$ and $\mathrm{Au}$, which both have approximately the same value of $\epsilon_{d}$.

A comparison between $G^{0} W^{0}$ hot-electron lifetimes in $\mathrm{Cu}$ and those determined from TR-TPPE experiments was presented in Ref. 13. At low hot-electron energies, the calculated lifetimes were found to be in agreement with the low- 


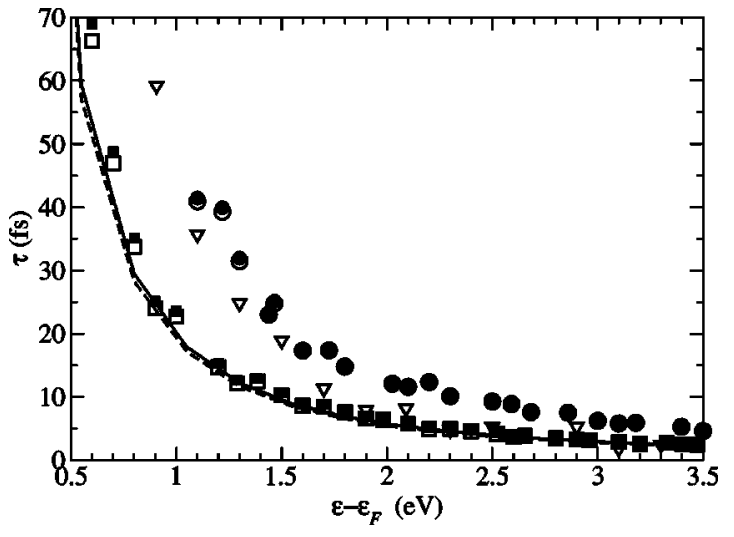

FIG. 4. As in Fig. 1, but for Ag. Here, solid and dashed lines represent $G W \Gamma$ and $G^{0} W^{0}$ lifetimes of hot electrons in a FEG with $r_{s}=3.02$, corresponding to the average density of $5 s^{1}$ electrons in Ag. Inverted triangles represent the TR-TPPE measurements reported in Ref. 34.

energy measurements of Knoesel et al. ${ }^{9}$ At larger energies, very good agreement was found with the lifetimes measured by Ogawa et al. ${ }^{6}$ at the (110) surface of $\mathrm{Cu}$, the only lowindex surface with no band gap for electrons emitted in the direction perperdincular to the surface. Our results indicate that the inclusion of exchange and correlation does not substantially change this agreement.

\section{B. Silver}

We have plotted in Fig. 4 our full first-principles $G W \Gamma$ calculation of the average lifetime $\tau(\varepsilon)$ of hot electrons in $\mathrm{Ag}$ (solid circles), as obtained in the ALDA from Eqs. (10)-(12) but with the inverse dielectric matrix $\epsilon_{\mathbf{G}, \mathbf{G}^{\prime}}^{-1}(\mathbf{q}, \omega)$ replaced by that of Eq. (19). For comparison, we also plot in the same figure our full first-principles $G^{0} W^{0}$ calculation (open circles), first-principles $G W \Gamma$ and $G^{0} W^{0} s$ calculations (solid and open squares), and $G W \Gamma$ and $G^{0} W^{0}$ FEG calculations with the electron density $n_{0}$ equal to that of $5 s^{1}$ electrons in $\mathrm{Ag}$ (solid and dashed lines). Also shown in this figure are the TR-2PPE measurements reported by Bauer and Aeschlimann $^{34}$ for the lifetime of hot electrons in a thin Ag film (inverted triangles).

Figure 4 shows that both in the presence (solid and open circles) and in the absence (solid and open squares) of $d$ states the overall impact of exchange and correlation on the lifetime of hot electrons in $\mathrm{Ag}$ is very small $(\sim 2 \%)$ in the whole hot-electron energy range under study. As occurs in the case of $\mathrm{Cu}$, the two separate sources of $\mathrm{XC}$ effects, which are both found to yield significant effects in the absence of $d$ states and considerably smaller effects in the presence of $d$ states, almost cancel each other. We also note from Fig. 4 that at the lowest hot-electron energies there is good agreement between our full $G^{0} W^{0}$ and $G W \Gamma$ calculations and the experimental data, which are both $\sim 2.5$ larger than in the absence of occupied $d$ states. At larger energies, however, our calculations lie slightly higher than the experimental curve.

At this point, it is interesting to notice that while hotelectron lifetimes in a FEG with $r_{s}=2.67$ (corresponding to

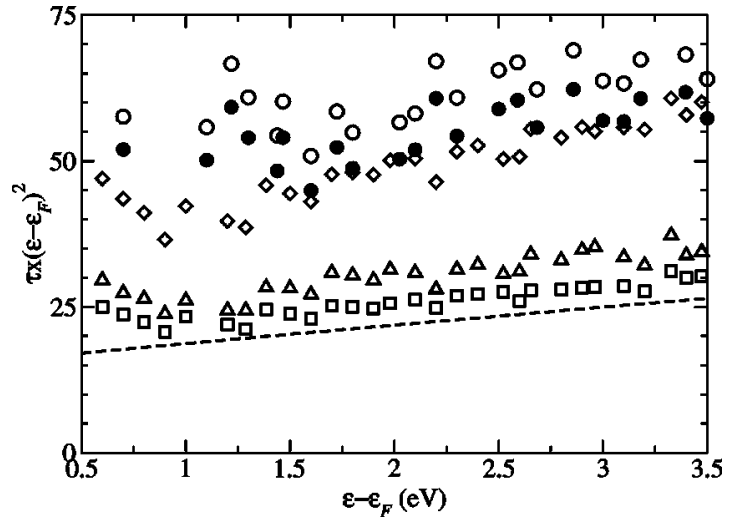

FIG. 5. As in Fig. 3, but for Ag. Solid circles now represent the full $G W \Gamma$ matricial calculation of Fig. 4, but now multiplied by $\left(\varepsilon-\varepsilon_{F}\right)^{2}$. The dashed line represents the $G^{0} W^{0}$ calculation for hot electrons in a FEG with $r_{s}=3.02$.

the average density of $4 s^{1}$ electrons in $\mathrm{Cu}$ ) are larger than in a FEG with $r_{s}=3.01$ (corresponding to the average density of $5 s^{1}$ electrons in $\mathrm{Ag}$ ), hot electrons are found to live slightly longer in $\mathrm{Ag}$ than in $\mathrm{Cu}$. Both in $\mathrm{Cu}$ and $\mathrm{Ag}$ first-pinciples $s$ calculations nearly coincide with their FEG counterparts, showing that band-structure effects are almost entirely due to the presence of $d$ electrons. Hence, the impact of $d$ electrons is found to be larger in $\mathrm{Ag}$ (with the onset of the $d$ band at $\sim 4 \mathrm{eV}$ below the Fermi level $\varepsilon_{F}$ ) than in $\mathrm{Cu}$ (with the onset of the $d$ band at $\sim 2 \mathrm{eV}$ below $\varepsilon_{F}$ ).

Figure 5 shows first-principles $G^{0} W^{0}$ calculations of the scaled lifetime $\tau(\varepsilon) \times\left(\varepsilon-\varepsilon_{F}\right)^{2}$ of hot electrons in Ag, as obtained from Eq. (15) by (i) assigning the $4 d$ shell to the core in the generation of the pseudopotential (squares), (ii) including all $4 d^{10}$ states only in the evaluation of $\operatorname{Im}\left[\epsilon_{\mathbf{G}, \mathbf{G}}(\mathbf{q}, \omega)\right]$ (triangles), (iii) including all $4 d^{10}$ states also in the evaluation of $\left|\epsilon_{\mathbf{G}, \mathbf{G}}(\mathbf{q}, \omega)\right|^{-2}$ (diamonds), and (iv) fully accounting for the presence of $4 d^{10}$ states (open circles). First of all, we note that it matters very little whether $d$ electrons are included in the evaluation of $\operatorname{Im}\left[\epsilon_{\mathbf{G}, \mathbf{G}}(\mathbf{q}, \omega)\right]$ or not. This is obviously due to the fact that in the case of $\mathrm{Ag}$ and at the hot-electron energies under study, $d$ electrons are too far below the Fermi level to participate in the creation of $e-h$ pairs. Nevertheless, it does not matter how far they are located below the Fermi level for them to participate in the screening of $e-e$ interactions. Indeed, Fig. 5 shows that the screening of $d$ electrons, which enters in the evaluation of $\left|\epsilon_{\mathbf{G}, \mathbf{G}}(\mathbf{q}, \omega)\right|^{-2}$, is responsible for the hot-electron lifetimes in Ag being $~ 2.5$ times larger than in the absence of occupied $d$ states.

\section{Gold}

Figure 6 shows our full first-principles $G W \Gamma$ calculation of the average lifetime $\tau(\varepsilon)$ of hot electrons in $\mathrm{Au}$ (solid circles), as obtained in the ALDA from Eqs. (10)-(12) but with the inverse dielectric matrix $\epsilon_{\mathbf{G}, \mathbf{G}^{\prime}}^{-1}(\mathbf{q}, \omega)$ replaced by that of Eq. (19). For comparison, we also plot in the same figure our full first-principles $G^{0} W^{0}$ calculation (open circles), first-principles $G W \Gamma$ and $G^{0} W^{0} s$ calculations (solid and open squares), and $G W \Gamma$ and $G^{0} W^{0}$ FEG calculations 


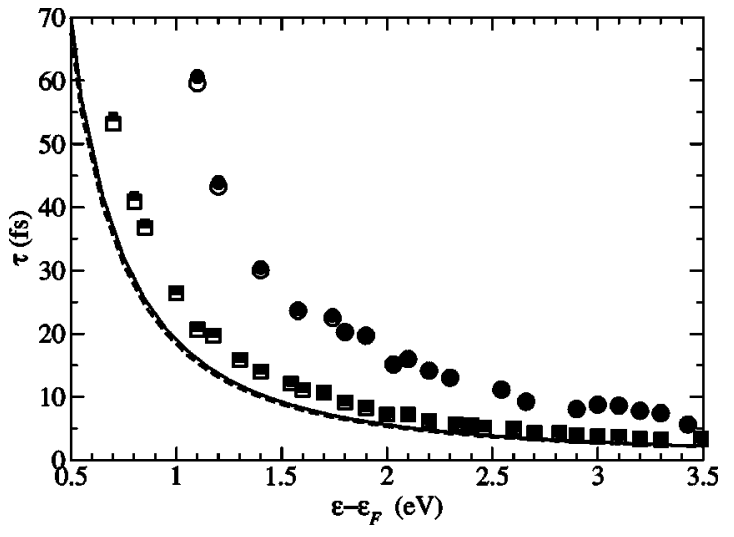

FIG. 6. As in Fig. 1, but for Au. Here, solid and dashed lines represent $G W \Gamma$ and $G^{0} W^{0}$ lifetimes of hot electrons in a FEG with $r_{s}=3.01$, corresponding to the average density of $6 s^{1}$ electrons in $\mathrm{Au}$.

with the electron density $n_{0}$ equal to that of $6 s^{1}$ electrons in $\mathrm{Au}$ (solid and dashed lines). The converged $G^{0} W^{0}$ calculation represented in Fig. 6 by open circles replaces the lifetimes reported in Ref. 17, which were too large by an overall factor of $\sim 1.4$.

As in the case of $\mathrm{Cu}$ and $\mathrm{Ag}$, the overall impact of exchange and correlation on the lifetime of hot electrons in $\mathrm{Au}$ is very small, now $\sim 2 \%$. We also note that our pseudopotential $G^{0} W^{0}$ calculations are in excellent agreement with the all-electron linearized augmented plane wave (LAPW) $G^{0} W^{0}$ calculations reported in Ref. 21, although they are $\sim 20 \%$ larger than the corresponding all-electron linear-muffin-tinorbital (LMTO) calculations reported in Ref. 18. As noted in Ref. 21, this discrepancy should be attributed to the atomicsphere approximation used in the LMTO calculations of Ref. 18. The LAPW $G^{0} W^{0}$ lifetimes of Ref. 21 were found to accurately reproduce the BEES spectra for the two prototypical $\mathrm{Au} / \mathrm{Si}$ and $\mathrm{Pd} / \mathrm{Si}$ systems, although they were approximately $40 \%$ shorter than the TR-2PPE measurements reported in Ref. 10. Our $G W \Gamma$ calculations indicate that the inclusion of exchange and correlation does not change these conclusions.

A closer analysis of our $G^{0} W^{0}$ calculations is presented in Fig. 7, where various calculations of the scaled lifetime $\tau(\varepsilon) \times\left(\varepsilon-\varepsilon_{F}\right)^{2}$ of hot electrons in $\mathrm{Au}$ is exhibited, as obtained from Eq. (15) by (i) assigning the $5 d$ shell to the core in the generation of the pseudopotential (squares), (ii) including $d$ states only in the evaluation of $\operatorname{Im}\left[\epsilon_{\mathbf{G}, \mathbf{G}}(\mathbf{q}, \omega)\right]$ (triangles), (iii) including $d$ states also in the evaluation of $\left|\epsilon_{\mathbf{G}, \mathbf{G}}(\mathbf{q}, \omega)\right|^{-2}$ (diamonds), and (iv) including $d$ states everywhere (open circles). As in the case of $\mathrm{Cu}$ and $\mathrm{Ag}$, these calculations show the major importance of the dynamical screening of $d$ electrons, which is responsible for the hotelectron lifetimes in $\mathrm{Au}$ being $~ 2.5$ times larger than in the absence of occupied $d$ states.

As noted in Ref. 17, the role that occupied $d$ states play in the screening of $e-e$ interactions is more important in $\mathrm{Au}$ than in $\mathrm{Cu}$, although the static polarizable background of $d$ electrons in these materials should be expected to be the same. However, our calculations indicate that $5 d$ bands in $\mathrm{Au}$ are more free-electron-like than $3 d$ bands in $\mathrm{Cu}$, thereby al-

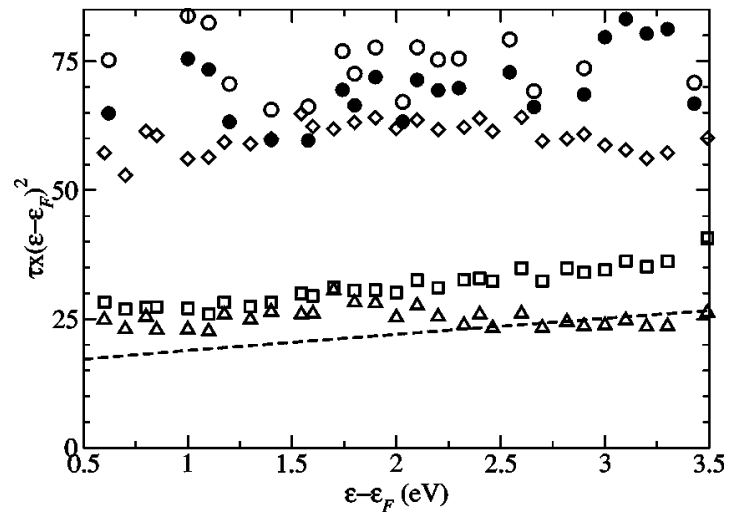

FIG. 7. As in Fig. 3, but for Au. Solid circles now represent the full $G W \Gamma$ matricial calculation of Fig. 6, but now multiplied by $\left(\varepsilon-\varepsilon_{F}\right)^{2}$. The dashed line represents the $G^{0} W^{0}$ calculation for hot electrons in a FEG with $r_{s}=3.01$.

lowing Au $5 d$ electrons to be more effective in the dynamical screening of $e-e$ interactions.

\section{SUMMARY AND CONCLUSIONS}

We have carried out extensive first-principles calculations of the inelastic lifetime of low-energy electrons in the noble metals $\mathrm{Cu}, \mathrm{Ag}$, and $\mathrm{Au}$, in the framework of the $G W \Gamma$ approximation of many-body theory. This approximation treats on the same footing $\mathrm{XC}$ effects between pairs of electrons within the Fermi sea (screening electrons) and between the excited hot electron and the Fermi sea. Our ALDA calculations indicate that the impact of exchange and correlation on either screening or the bare interaction of the hot electron with the Fermi sea, which is significant in the absence of $d$ states, is considerably reduced in the presence of $d$ states. Moreover, these small xc contributions have opposite signs and it is the latter which dominates, leading to $G W \Gamma$ lifetimes that are larger than their $G^{0} W^{0}$ counterparts by $\sim 3 \%$ in $\mathrm{Cu}$ and $\sim 2 \%$ in $\mathrm{Ag}$ and $\mathrm{Au}$.

We have established the role that occupied $d$ states play in the relaxation of hot electrons in the noble metals. We have found that deviations from the hot-electron lifetimes in these materials from those of hot electrons in the corresponding free gas of valence $s p$ electrons are mainly due to the participation of occupied $d$ states in the screening of $e-e$ interactions, no matter whether occupied $d$ states can participate (as occurs in $\mathrm{Cu}$ and $\mathrm{Au}$ ) or not (as occurs in $\mathrm{Ag}$ ) in the creation of $e-h$ pairs. Au $5 d$ electrons lie further away from the nuclei than $\mathrm{Cu} 3 d$ electrons, thereby occupied $d$ states in $\mathrm{Au}$ being more capable to screen the $e$ - $e$ interactions than in $\mathrm{Cu}$. The dynamical screening of $d$ electrons yields lifetimes of hot electrons that are larger than in the absence of $d$ states by a factor of $\sim 2$ in the case of $\mathrm{Cu}$, and by a factor of $\sim 2.5$ in the case of $\mathrm{Ag}$ and $\mathrm{Au}$.

We have found that our $G^{0} W^{0}$ calculations of the hotelectron lifetimes in $\mathrm{Au}$ are in excellent agreement with the corresponding all-electron LAPW calculations reported in Ref. 21, which gives us confidence in the accuracy of our pseudopotential calculations. Our $G^{0} W^{0}$ lifetimes are found 
to be $\sim 20 \%$ larger than the corresponding all-electron LMTO calculations reported in Ref. 18, which should be attributed to the atomic-sphere approximation used within the LMTO scheme. We also note that our $G^{0} W^{0}$ lifetimes of hot electrons in the noble metals are systematically higher than those reported in Ref. 16, especially at the lowest energies. These authors obtained the lifetime broadening as the full width at half maximum of the so-called spectral function, which they calculated in the $G^{0} W^{0}$ approximation. Apart from a renormalization factor which accounts for the deviation of the hot-electron energy from its noninteracting counterpart and which increases the lifetime by a factor of $\sim 20 \%$, the lifetimes obtained in this way should agree with our $G^{0} W^{0}$ calculations.

Since our $G W \Gamma$ calculations are very close to their $G^{0} W^{0}$ counterparts, the inclusion of exchange and correlation does not substantially change the comparison between $G^{0} W^{0}$ calculations and TR-2PPE and BEES measurements existing in the literature for $\mathrm{Cu}$ and $\mathrm{Au}$. In the case of $\mathrm{Ag}$, we have compared our new $G^{0} W^{0}$ and $G W \Gamma$ calculations with the recent experimental TR-2PPE measurements reported in Ref. 34. At the lowest hot-electron energies there is good agreement between our calculations and the experimental data. At larger energies, our calculations are found to lie slightly higher than the experimental curve.

\section{ACKNOWLEDGMENTS}

We acknowledge partial support by the University of the Basque Country, the Basque Hezkuntza, Unibertsitate eta Ikerketa Saila, the Spanish Ministerio de Ciencia y Tecnología, and the Max Planck Research Funds.
${ }^{1}$ J. J. Quinn and R. A. Ferrel, Phys. Rev. 112, 812 (1958).

${ }^{2}$ R. H. Ritchie, Phys. Rev. 114, 644 (1959).

${ }^{3}$ P. M. Echenique, J. M. Pitarke, E. V. Chulkov, and A. Rubio, Chem. Phys. 251, 1 (2000).

${ }^{4}$ C. A. Schmutenmaer, M. Aeschlimann, H. E. Elsayed-Ali, R. J. D. Miller, D. A. Mantell, J. Cao, and Y. Gao, Phys. Rev. B 50, 8957 (1994).

${ }^{5}$ T. Hertel, E. Knoesel, M. Wolf, and G. Ertl, Phys. Rev. Lett. 76, 535 (1996)

${ }^{6}$ S. Ogawa, H. Nagano, and H. Petek, Phys. Rev. B 55, 1 (1997).

${ }^{7}$ J. Cao, Y. Gao, R. J. D. Miller, H. E. Elsayed-Ali, and D. A. Mantell, Phys. Rev. B 56, 1099 (1997).

${ }^{8}$ M. Aeschlimann, M. Bauer, S. Pawlik, R. B. W. Weber, D. Oberli, and H. C. Siegmann, Phys. Rev. Lett. 79, 5158 (1997).

${ }^{9}$ E. Knoesel, A. Hotzel, and M. Wolf, Phys. Rev. B 57, 12812 (1998).

${ }^{10}$ J. Cao, Y. Gao, H. E. Elsayed-Ali, R. J. D. Miller, and D. A. Mantell, Phys. Rev. B 58, 10948 (1998).

${ }^{11}$ H. Petek and S. Ogawa, Prog. Surf. Sci. 56, 239 (1998).

${ }^{12}$ K. Reuter, U. Hohenester, P. de Andrés, F. J. García-Vidal, F. Flores, K. Heinz, and P. Kocevar, Phys. Rev. B 61, 4522 (2000).

${ }^{13}$ I. Campillo, J. M. Pitarke, A. Rubio, E. Zarate, and P. M. Echenique, Phys. Rev. Lett. 83, 2230 (1999).

${ }^{14}$ W.-D. Schöne, R. Keyling, M. Bandić, and W. Ekardt, Phys. Rev. B 60, 8616 (1999).

${ }^{15}$ I. Campillo, V. M. Silkin, J. M. Pitarke, E. V. Chulkov, A. Rubio, and P. M. Echenique, Phys. Rev. B 61, 13484 (2000).

${ }^{16}$ R. Keyling, W.-D. Schöne, and W. Ekardt, Phys. Rev. B 61, 1670 (2000)

${ }^{17}$ I. Campillo, J. M. Pitarke, A. Rubio, and P. M. Echenique, Phys. Rev. B 62, 1500 (2000).
${ }^{18}$ V. P. Zhukov, F. Aryasetiawan, E. V. Chulkov, I. G. Gurtubay, and P. M. Echenique, Phys. Rev. B 64, 195122 (2001).

${ }^{19}$ V. P. Zhukov, F. Aryasetiawan, E. V. Chulkov, and P. M. Echenique, Phys. Rev. B 65, 115116 (2002).

${ }^{20}$ M. R. Bacelar, W.-D. Schöne, R. Keyiling, and W. Ekardt, Phys. Rev. B 65, 115116 (2002).

${ }^{21}$ F. Ladstädter, P. de Pablos, U. Hohenester, P. Pusching, C. Ambrosch-Draxl, P. de Andrés, F. J. García-Vidal, and F. Flores, Phys. Rev. B 68, 085107 (2003).

${ }^{22}$ L. Hedin and S. Lundqvist, Solid State Phys. 23, 1 (1969).

${ }^{23}$ G. D. Mahan and B. Sernelius, Phys. Rev. Lett. 62, 2718 (1989).

${ }^{24}$ G. D. Mahan, Many-Particle Physics (Plenum, New York, 1990).

${ }^{25}$ P. Hohenberg and W. Kohn, Phys. Rev. 136, B864 (1964).

${ }^{26}$ W. Kohn and L. Sham, Phys. Rev. 140, A11333 (1965).

${ }^{27}$ N. Trouiller and J. L. Martins, Phys. Rev. B 43, 1993 (1991).

${ }^{28}$ E. Runge and E. K. U. Gross, Phys. Rev. Lett. 52, 997 (1984).

${ }^{29}$ M. Petersilka, U. J. Gossmann, and E. K. U. Gross, Phys. Rev. Lett. 76, 1212 (1996).

${ }^{30}$ J. Perdew and A. Zunger, Phys. Rev. B 23, 5048 (1981).

${ }^{31}$ D. M. Ceperly and B. J. Alder, Phys. Rev. Lett. 45, 1196 (1980).

${ }^{32}$ J. J. Quinn, Appl. Phys. Lett. 126, 1453 (1963).

${ }^{33}$ P. B. Johnson and R. W. Christy, Phys. Rev. B 6, 4370 (1972).

${ }^{34}$ M. Bauer and M. Aeschlimann, J. Electron Spectrosc. Relat. Phenom. 124, 225 (2002).

${ }^{35}$ This is the often-called $G^{0} W$-RPA.

${ }^{36}$ Introduction of this XC kernel into Eq. (4) yields the so-called $G^{0} W$-ALDA.

${ }^{37} \mathrm{For} \mathrm{Cu}$, we keep $\sim 900$ plane waves in the expansion of each Bloch state. In the case of $\mathrm{Ag}$ and $\mathrm{Au}$, we keep $\sim 1300$ plane waves. 